\title{
Community care of people with late stage HIV infection
}

\author{
R J D George
}

With the emergence of HIV infection as a major social and medical threat, many areas of medical practice and social attitude have required fundamental review. This editorial looks at some of the issues raised by HIV infection in the care of infected individuals in the community, particularly at the end of their life.

With the emergence of HIV and AIDS in the early 1980 s, the medical and caring professions were confronted with an illness that presented frequently in an acute and catastrophic way with overwhelming opportunistic infection requiring hospital admission. In this group of young patients their only contact with medical services hitherto has tended to be the genitourinary clinics or casualty departments. Prior to the development of HIV related disease, few seropositive patients have required their general practitioners.

With the limited knowledge and understanding of the disease processes associated with HIV, and the frequent need for admission to hospital, medical care has tended to stay with and be run by hospital and outpatient based services. However, with the passage of time and improvement in therapeutics, we have been faced with an increased number of people with varying levels of clinical and physical disability and a limited life expectancy who have the desire and potential to spend much of their time at home.

Long term, it only makes sense to consider community care for HIV alongside existing services for other chronic or terminal diseases. In broad terms, these fall into three groups:

(1) The "acute sector" both in and outpatient for sophisticated diagnostics and treatment of acute episodes. Philosophically, they remain fairly separate from the community. Patients see hospital based clinical staff, are required to attend clinics, but may also see staff such as health advisors, social workers, community liaison staff etc who are able to extend or develop services based at home.

University College and Middlesex School of Medicine, Community Care Team, National Temperance Hospital, Hampstead Road, London NW1 2LT, UK

R J D George
(2) Neighbourhood services. The bedrock of community-based NHS services, in the UK at least, remains the primary health-care team (PHCT), comprising general practitioner, practice nurse, community nursing, physiotherapy etc. In theory, all patients should be registered with a practice, although the young, or those who have had poor experience of general practice (many of those diagnosed as HIV positive) are reticent to avail themselves of this service. The local authorities provide social work, home-help, welfare benefits and in some circumstances occupational therapy and other support services. Patients have rarely had contact with these services unless they have had social or practical needs related to ill health.

(3) The specialist services for HIV have almost all found their birth in the voluntary sector. ${ }^{1}$ These fall really into two groups:

Residential services. Although the situation is changing, these are only developed to any degree in metropolitan areas and in London in particular where AIDS dedicated respite and hospice facilities exist for example at the Mildmay Mission Hospital in the East End and at the London Lighthouse in the West End. A number of projects have also developed piecemeal to cater for people needing various levels of sheltered accommodation.

Domiciliary voluntary services have formed an essential and integral part of HIV services up until now, both in the speed with which they were established in the early mid-eighties, and their flexibility. They vary enormously across the country. Information on local provision is best sought from the local AIDS coordinator.

Whilst traditionally the basic responsibility for medical services for people with any illness has resided with the general practitioner and primary health care team, in the case of HIV, this has not been the case for a number of reasons:

Confidentiality. Apart from the small number of iatrogenic HIV infections from contaminated blood products, the vast majority of people have acquired the disease sexually, or within the drug using community by sharing needles and syringes. Historically, those with STDs have attended the open access 
clinics, frequently outside their own health district, without having to involve their family practitioners. For individuals who are otherwise healthy, and who have had treatable or short lived problems, such a system has worked very well. However, in patients with long term or chronic disease it has significant limitations. Drug users have always been alienated from conventional medical services. Because of the social stigma of HIV, many people have been extremely reluctant to inform their general practitioner of the disease. Unfortunately these anxieties have been borne out by studies suggesting that confidentiality may not be safeguarded as patients would hope. ${ }^{23}$ Certainly the predominant reason given for patients not using general practitioners is concern about confidentiality. ${ }^{4}$

Non-familiarity with the disease. The immunodeficiency that follows HIV infection leads frequently to changing pathologies involving a variety of systems simultaneously, and this may be bewildering to the non-specialist. As many patients are extremely well informed about their disease, they have a low level of confidence in non-specialists. Prior to their diagnosis with AIDS many have been perfectly fit and well and had no contact with their general practitioners. The professionals with whom the patients have established relationship are those within the STD clinics, with whom they may have consulted over several years prior to diagnosis. Naturally there is a reticence to extend this small sphere of carers.

This absence of familiarity or trust of the general practitioner often compounds any anxiety about confidentiality and knowledge. Not surprisingly, studies on the knowledge of general practitioners seem to show a wide spectrum with hands on experience being the most significant determinant of awareness of social, educational and clinical issues. $^{235}$

Open access clinics. The gay community in particular has tended to polarise around services that have been sympathetic to them at a time when society has been hostile. In the early and mid 1980 s, when the disease first emerged amongst the gay community, such hospitals had rapid exposure to the diversity of clinical problems by "natural selection". Frequently patients had travelled considerable distances to attend these clinics. With the development of chronic or recurrent illness, they had a natural reluctancy to move to local services or involve their general practitioners unless absolutely necessary. This, to a degree, has been reinforced by clinicians in the centres, as a rapid development of expertise has depended upon a large patient load, and there has been a degree of symbiosis.

However, as the epidemic has developed and there has been an increasingly large group of patients with late stage disease and significant progressive disability, the problems of providing adequate and appropriate care within the community is a priority. Significant management problems have emerged:

(1) Patients need rapid access to services, particularly as opportunistic infections may develop quickly. Whilst dissemination of knowledge has been good, research and investigation of new treatments has been based around the established HIV centres. For patients living at a distance from their chosen hospitals, this had led to difficulties in travel or alternatively to prolonged admissions into hospital beds.

(2) As with all chronic and progressive disease, needs are by no means confined to clinical management. In HIV, dietary needs and the issues of malnutrition have been prominent in patients at all stages of their illness, ${ }^{6}$ with virtually all patients needing specialist advice at some stage. In our experience at the Middlesex and University College Hospitals, $88 \%$ of patients with late stage disease need ongoing nutritional care. Physical disability, and particularly the emergence of neurological deficit and impaired vision has meant that occupational therapy plays a vital role, $66 \%$ of patients need the services of an OT in the late stages of disease. This has placed additional burdens on already badly stretched community services. Practical support is a universal need and may not be available from family or friends. Whilst the voluntary sector has gone a long way to meet the shortfall, these have remained significant problems. Furthermore, personal provision for unexpected disability by the young is usually very low priority and is compounded by the difficulties of getting adequate insurance cover. As examples, patients may have an acute need for financial planning, welfare benefits or accommodating unexpected social deprivation and estate management in the face of encephalopathy or provision for early retirement. All need expert advice and may be a major source of stress.

(3) The counselling needs of individuals with HIV are well known. ${ }^{7}$ However, in late stage disease, patients suffer particular personal "bereavements"; the loss of health, appearance, employment etc, and ultimately in preparing for death several decades earlier than expected. There are also particular problems for bereaved "relatives", the most significant and pervasive being the self-enforced isolation of many who feel unable to be honest about the cause of bereavement from what still remains a socially unacceptable disease.

In trying to find ways of developing community based care for people with HIV, a service provided must account for the needs outlined above. It is inefficient and illogical to provide a service that renders the Primary Health Care Team redundant as 
epidemiology is suggesting that most practitioners will ultimately come in contact with HIV infection. ${ }^{8}$ There must therefore be a mechanism of liaison, coordination and facilitation which takes account of confidentiality, promotes education and training of community based staff and wherever possible enables local services to assimilate the management of HIV into generic services. Provision must also involve the informal care network around an individual.

This has been solved in a variety of ways by different centres, for example by the provision of liaison staff or facilitators in line with the recommendations of the Oxfordshire study. ${ }^{9}$ Other centres have used peripatetic specialist teams to assist the PHCTs on site using patient contact and shared care as a vehicle for involvement and education. ${ }^{1011}$ Studies certainly suggest that direct exposure to patients considerably enhances the confidence and ability of general practitioners to deal with HIV. ${ }^{134}$

The teaching hospitals within Bloomsbury have a large clinical load. This has justified the establishment of a multidisciplinary community based team which gives patients and carers and the primary health care team rapid access to medical and nursing expertise, occupational therapy and dietetics. Where appropriate, the team provides a channel to link and integrate the hospital based clinical services including social work and counselling, and also the specialist services that cater for the specific needs of drug users.

The remit of the services has been to promote and facilitate community based care, particularly for people with late or end stage HIV related disease (the median time of a person under care is 12 weeks, range 1-52 weeks) much as the terminal care services that have developed for cancer. Apart from symptom control and clinical advice, care extends to emotional and pastoral care of both patient and loved ones and extends to bereavement follow-up where needed.

The team's effectiveness has been particularly evident in the involvement of the PHCTs in the care of people at home. In the three years since its inception, involvement by general practitioners in about $30 \%$ of cases has now risen to almost $90 \% .{ }^{12}$ In the last three months of life, patients spend on average two thirds of their time at home, with the remaining third divided equally between hospice and hospital. The presence of the team is felt to have contributed in part to the falling ratio of hospital beds occupied to AIDS caseload at the Middlesex Hospital. ${ }^{13}$ Auditing the last 150 deaths, approximately $40 \%$ have occurred at home, $40 \%$ in hospice and $20 \%$ in hospital (unpublished). The statistics are similar to those found amongst the terminal care teams caring for cancer, although less deaths still occur at home. ${ }^{14}$

\section{Conclusions}

It is possible using community care teams similar to those developed by the hospice movement to educate and involve PHCTs in the care of people with late stage HIV infection at home. This is borne out by the experience of other specialist teams. ${ }^{112}$ Continued development along these lines is essential as HIV infection passes into the heterosexual community and falls within the experience of most practitioners in urban areas. ${ }^{815} 16$ Many health districts are responding by expanding or developing new initiatives to provide tailored local based services. These will, of course, take on character appropriate to the populations being served-in large conurbations, there may be a place for developing appropriate specialist services; in other places, existing services may need education or modest expansion. Suffice it to say that in the referral centres and areas where there are high densities of HIV infected people, specialist services will probably have a place as many people will continue to choose, to base their care with specialist centres. How the implementation of the White Paper and Community Care Bills will influence these developments remains to be seen.

1 Bould M, Peacock G (eds). AIDS Models of Care. King's Fund, London Boroughs Training Committee, Terrence Higgins Trust \& Frontliners. King's Fund Centre 1989.

2 Naji SA, Russell IT, Foy CJW, et al. HIV Infection and Scottish general practice: knowledge and attitudes. J R Coll Gen Pract 1989;39:284-8.

3 Boyd JS, Kerr S, Maw RD, et al. Knowledge of HIV infection and AIDS, and attitudes to testing and counselling among general practitioners in Northern Ireland. $\mathrm{Br} J$ General Practice 1990;40:158-60.

4 Mansfield SJ, Singh S. The general practitioner and human immunodeficiency infection: an insight into patient's attitudes. $J R$ Coll Gen Pract 1989;39:103-5.

5 King MB. AIDS and the general practitioner: views of patients with HIV infection and AIDS. $B M J 1988 ; 297: 182-4$.

6 Peck K, Johnson S. The role of nutrition in HIV infection. $J$ Human Nutrition $\mathcal{E}$ Diet 1990;3:147-57.

7 Miller D. Living with AIDS. London: MacMillan 1988.

8 Day NE. Communicable Diseases Report. Acquired Immune Deficiency Syndrome in England and Wales to end 1993. Report of the working group convened by the Director of the Public Health Laboratory Service. Colindale, PHLS Communicable Disease Surveillance Centre, 1990.

9 Anderson P, Mayon-White R. General Practitioners and management of infection with HIV. BMJ 1988;296:535-7.

10 George RJD, Hart G. The Bloomsbury Response. In: Responding to the AIDS Challenge. Pye M, Kapila M, Buckley G, Cunningham D, eds. Health Education Authority, Harlow. Longman 1989.

11 Smits A, Mansfield S, Singh S. Facilitating care of patients with HIV infection by hospital and primary care teams. BMJ 1990;300:241-3.

12 Butters E, Higginson I, George RJD, et al. Two community HIV/AIDS teams: referrals, patient characteristics and patterns of care. Health Trends 1990 . (In press).

13 Shergold C, Johnson AM, Walter C, Adler MW. Patterns of hospital care for inpatients with HIV infection. Soc Med Annual Scientific Meeting (Abstr) 1990.

14 Higginson I, McCarthy M. Measuring symptoms in terminal cancer: are pain and dyspnoea controlled? J $R$ Soc Med 1989;82:264-7.

15 Boyton R, Scrambler G. Survey of general practitioners' attitudes to AIDS in the North West Thames and East Anglian Regions. BMJ 1988:296:538-40.

16 Gallagher M, Foy C, Rhodes T, et al. HIV infection and AIDS in England and Wales: general practitioners' workload and contact with patients. Br J General Practice 1990;40:154-7.

Accepted for publication 19 March 1991 